\title{
Performance des définitions administratives de cas pour les affections concomitantes de la sclérose en plaques au Manitoba et en Nouvelle-Écosse
}

\author{
R. A. Marrie, M.D., Ph. D. (1, 2); J. D. Fisk, Ph. D. (3, 4, 5); K. J. Stadnyk, M. Sc. (5); H. Tremlett, Ph. D. (6); \\ C. Wolfson, Ph. D. (7, 8); S. Warren, Ph. D. (9); V. Bhan, M.D. (3, 5); B. N. Yu, M.D., Ph. D. (1, 2), pour l'équipe des IRSC \\ dans le cadre du projet sur l'épidémiologie et l'effet des maladies concomitantes sur la sclérose en plaques
}

Cet article a fait l'objet d'une évaluation par les pairs.

\section{Résumé}

Introduction : Au fur et à mesure du vieillissement de la population et de l'augmentation de la prévalence d'affections concomitantes, le recours à des méthodes fiables et efficaces de surveillance des affections concomitantes de maladies chroniques telles que la sclérose en plaques (SP) s'avère de plus en plus nécessaire.

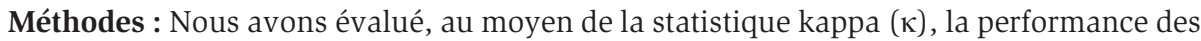
définitions administratives de cas pour les affections concomitantes fréquemment observées en lien avec la SP en comparant les concordances entre les données administratives et les données provenant d'autodéclarations au Manitoba (MB) $(n=606)$ et en Nouvelle-Écosse (NS) ( $n=1923$ ).

Résultats : Les concordances entre les définitions administratives et les autodéclarations étaient bonnes pour l'hypertension $(\kappa=0,69[\mathrm{NS}]$ et $0,76[\mathrm{MB}])$ et le diabète $(\kappa=0,70$ [NS] et 0,66 [MB]), modérées pour l'hyperlipidémie ( $\kappa=0,53$ [NS] et 0,51 [MB]) et la cardiopathie ( $\kappa=0,42[\mathrm{NS}]$ et $0,51[\mathrm{MB}])$ et médiocres pour l'anxiété $(\kappa=0,27$ [NS] et $0,26[\mathrm{MB}])$. La concordance était bonne en Nouvelle-Écosse pour la maladie inflammatoire chronique de l'intestin $(\kappa=0,71)$ et modérée pour l'épilepsie $(\kappa=0,48)$.

Conclusion : Les définitions administratives étaient performantes dans les deux provinces pour plusieurs affections concomitantes fréquemment observées en lien avec la SP. À la lumière de ces résultats, il semble que ces définitions puissent être utilisées plus largement au Canada et dans les études nationales.

Mots-clés : Données administratives, validation, affections concomitantes, sclérose en plaques

\section{Introduction}

Les affections concomitantes sont associées pour bon nombre de maladies chroniques à une série de conséquences négatives, notamment des taux accrus d'incapacité, de mortalité et d'utilisation des services de santé ${ }^{1}$. Par exemple, dans le cas de la maladie d'Alzheimer, les affections concomitantes de nature vasculaire, telle l'hypertension, sont associées à une perte plus rapide des facultés cognitives $^{2}$. La sclérose en plaques (SP) est une maladie neurologique chronique dont souffrent plus de deux millions de personnes sur la planète ${ }^{3}$. Au Canada, l'incidence et la prévalence de cette maladie sont élevées ${ }^{4}$, les taux annuels d'incidence pouvant atteindre 23,9 pour 100000 habitants $^{5}$ et le taux brut estimatif de prévalence se chiffrant en 2000-2001 à 240 pour 100000 habitants $^{6}$. Les affections concomitantes physiques comme mentales sont fréquentes chez les personnes atteintes de SP. La présence de ces affections peut retarder le diagnostic et entraîner des taux accrus d'incapacité et

Rattachement des auteurs :

1. Département de médecine interne, Université du Manitoba, Winnipeg (Manitoba), Canada

2. Département des sciences de la santé communautaire, Université du Manitoba, Winnipeg (Manitoba), Canada

3. Département de médecine, Université Dalhousie, Halifax (Nouvelle-Écosse), Canada

4. Département de psychiatrie, Université Dalhousie, Halifax (Nouvelle-Écosse), Canada

5. Régie de la santé du district de la capitale, Halifax (Nouvelle-Écosse), Canada

6. Département de médecine (neurologie), Université de la Colombie-Britannique, Vancouver (Colombie-Britannique), Canada

7. Département d'épidémiologie, de biostatistiques et de santé au travail, Université McGill, Montréal (Québec), Canada

8. Institut de recherche du Centre universitaire de santé McGill, Montréal (Québec), Canada

9. Faculté de médecine de réadaptation, Université de l'Alberta, Edmonton (Alberta), Canada

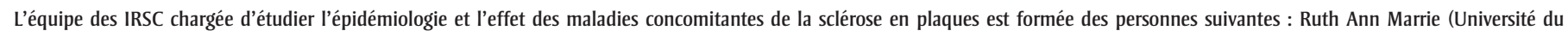

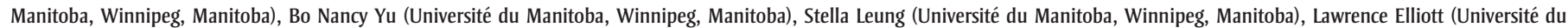

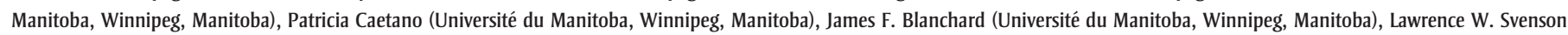

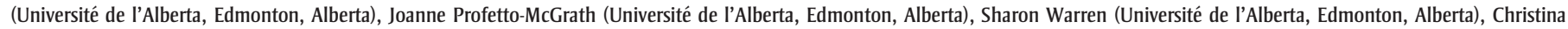

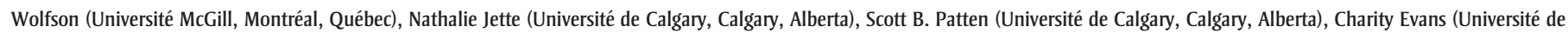

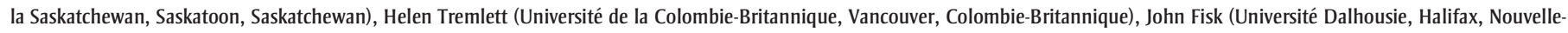
Écosse), Virender Bhan (Université Dalhousie, Halifax, Nouvelle-Écosse) et Michelle Ploughman (Université Mémorial, St. John's, Terre-Neuve).

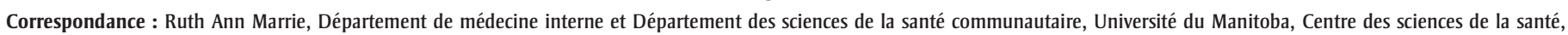
GF-533, 820, rue Sherbrooke, Winnipeg (Manitoba) R3A 1R9; tél. : 204-787-4951; téléc. : 204-787-1486; courriel : rmarrie@hsc.mb.ca 
une diminution de la qualité de vie $\mathrm{e}^{7-10}$. Pourtant, et malgré le besoin impératif de mieux comprendre l'effet de ces affections concomitantes, il existe relativement peu de méthodes fiables et efficaces pour les mesurer dans des contextes variés ${ }^{11}$. Ceci est particulièrement vrai à l'échelle de la population, où l'on a besoin d'évaluer l'effet des affections concomitantes de la SP sur les services de santé (hospitalisations, consultations médicales, soins préventifs et leur accès, etc.) et sur d'autres éléments mesurables à cette échelle, comme la mortalité ${ }^{12}$.

Parmi les sources de données permettant de repérer la présence d'affections concomitantes dans une population, on trouve les dossiers médicaux, les données provenant d'enquêtes (entrevues ou autodéclarations) et les données administratives (demandes de remboursement de services de santé) ${ }^{13}$. Aucune source n'est cependant idéale pour tous les plans d'étude, ou même pour toutes les affections concomitantes ${ }^{14}$. Dans certains pays, dont le Canada, les données administratives constituent une option intéressante pour réaliser des recherches sur les affections concomitantes à l'échelle de la population, car elles offrent un bon rapport coût-efficacité ${ }^{15}$. Ce type de données a été utilisé par exemple avec succès pour la surveillance nationale de maladies chroniques comme le diabète ${ }^{16}$. Les définitions administratives de cas ont été validées aussi pour l'analyse de plusieurs affections concomitantes tant physiques que mentales observées chez des personnes atteintes de SP à l'échelle d'une province canadienne, le Manitoba ${ }^{17-20}$. Les caractéristiques des bases de données sur les demandes de remboursement soumises par les médecins ainsi que sur les pratiques de facturation varient cependant d'une province à l'autre ${ }^{21}$. Sont variables par exemple le nombre de champs diagnostiques permis dans les demandes de remboursement des médecins et la spécificité des codes diagnostiques en fonction du nombre de chiffres de ces codes, deux caractéristiques susceptibles d'influer à la fois sur la sensibilité et sur la spécificité des définitions de cas. Il faut donc évaluer la performance de ces méthodes dans d'autres provinces, surtout si l'on veut comparer les résultats fondés sur ces méthodes entre provinces ou territoires.

Dans cette étude, nous avons comparé les performances des définitions administratives de cas élaborées pour la population atteinte de SP dans deux provinces canadiennes, le Manitoba et la Nouvelle-Écosse, afin de mettre au point des méthodes valables pour l'étude des affections concomitantes touchant l'ensemble de la population canadienne atteinte de SP.

\section{Méthodologie}

Nous avons procédé pour cette étude de validation à l'analyse secondaire de données cliniques et de données administratives couplées provenant du Manitoba et de la Nouvelle-Écosse. Nous présentons d'abord les méthodes utilisées au Manitoba puis celles employées en Nouvelle-Écosse.

\section{Manitoba}

\section{Contexte}

Le Manitoba affiche une population stable de près de 1,2 million d'habitants ${ }^{22}$. Les données ont été extraites des demandes de remboursement de services de santé de la province effectuées entre 2001 et 2006 et des autodéclarations de 604 patients atteints de SP portant sur l'ensemble de leur vie jusqu'en 2006. Le comité d'éthique de la recherche de l'Université du Manitoba a donné son approbation pour cette portion de l'étude et c'est le comité de protection des renseignements personnels de Santé Manitoba qui a accordé la sienne pour l'accès aux données administratives.

\section{Données administratives}

Santé Manitoba est le ministère provincial responsable de la prestation des soins de santé pour $98 \%$ de la population du Manitoba $^{22}$. Santé Manitoba tient à jour des dossiers informatisés pour toutes les demandes de remboursement de services de santé, notamment les demandes soumises par les hôpitaux et par les médecins. Ces demandes contiennent le numéro d'identification médical personnel (NIMP) du résidant qui a reçu le service. Dans le registre de population sont inscrits le sexe, les dates de naissance et de décès ainsi que les dates de couverture par l'assurance maladie provinciale. La base de données sur les congés des patients comprend le NIMP, les dates d'admission et de congé et jusqu'à 16 codes diagnostiques. Les diagnostics ont été consignés au moyen des codes à 5 chiffres de la Classification internationale des maladies (CIM)-9 jusqu'en 2004 et ils le sont depuis à l'aide des codes de la CIM-10-CA. Nous nous sommes servis de tous les diagnostics inscrits dans la base de données sur les congés des patients. Chaque demande de remboursement d'un médecin comporte le NIMP du patient et un code à 3 chiffres de la CIM-9 correspondant à un diagnostic précis effectué par ce médecin. Pour des raisons de confidentialité, le couplage a été effectué dans cette étude au moyen de NIMP brouillés, à partir de versions anonymisées des bases de données administratives fournies par Santé Manitoba (voir ci-dessous).

\section{Définitions administratives de cas des affections concomitantes}

Les définitions de cas utilisées dans cette étude ont été élaborées et validées auparavant au Manitoba à l'aide d'une cohorte de personnes atteintes de SP (voir tableau 1) ${ }^{17-19,23}$. En bref, nous avons généré des listes de codes des CIM-9 et 10 correspondant aux termes cliniques décrivant les affections concomitantes sélectionnées, celles-ci ayant été choisies en fonction de l'importance qu'on leur accordait dans le cadre des soins liés à la SP. Il s'agissait soit de celles qui avaient été signalées par plus de $5 \%$ des patients atteints de SP, soit de celles décrites dans la littérature comme ayant un lien avec les résultats cliniques ${ }^{7-10,24}$. Nous avons élaboré plusieurs définitions de cas pour chaque affection, en faisant varier le nombre nécessaire de demandes de remboursement par les hôpitaux et par les médecins ainsi que le nombre d'années de données utilisées pour déterminer si le patient en était atteint ${ }^{25}$.

\section{Cohorte de validation du Manitoba}

Nous avons comparé la classification des affections concomitantes reposant sur des définitions administratives de cas et celle reposant sur les diagnostics d'affections concomitantes reposant sur une norme de référence reçus par 604 patients atteints de maladie démyélinisante, dans le but d'optimiser la concordance mesurée par la valeur de la statistique kappa ( $\kappa$ ) entre ces 
TABLEAU 1

Définitions administratives de cas (demandes de remboursement de services de santé) utilisées pour identifier les affections concomitantes au sein de la population atteinte de sclérose en plaques, Nouvelle-Écosse et Manitoba (Canada)

\begin{tabular}{|c|c|c|c|c|}
\hline Affection concomitante & Codes de la CIM-9 & Codes de la CIM-10 & $\begin{array}{l}\text { Nombre d'années } \\
\text { de données }\end{array}$ & $\begin{array}{l}\text { Nombre et type de demandes de rembour- } \\
\text { sement d'hôpitaux ou de médecins }{ }^{\mathrm{a}}\end{array}$ \\
\hline Hypertension & 401 à 405 & I10 à I13, I15 & 2 & $\geq 1 \mathrm{H}$ ou $\geq 2 \mathrm{M}$ \\
\hline Hyperlipidémie & 272 & E780, E782, E784, E785 & 5 & $\geq 1 \mathrm{H}$ ou $\geq 2 \mathrm{M}$ \\
\hline Diabète & 250 & E10 à E14 & 5 & $\geq 1 \mathrm{H}$ ou $\geq 2 \mathrm{M}$ \\
\hline Cardiopathie & 410 à 414 & I20 à I25 & 5 & $\geq 1 \mathrm{H}$ ou $\geq 2 \mathrm{M}$ \\
\hline $\begin{array}{l}\text { Maladie inflammatoire } \\
\text { chronique de l'intestin }\end{array}$ & 555,556 & K50, K51 & & $\begin{array}{l}\geq 5 \mathrm{H} \text { ou } \mathrm{M} \text { ou (si résidant dans la province } \\
\text { depuis moins de } 2 \text { ans) } \geq 3 \mathrm{H} \text { ou } \mathrm{M}\end{array}$ \\
\hline Épilepsie & 345 & G40, G41 & 3 & $\geq 1 \mathrm{H}$ ou $\geq 2 \mathrm{M}$ \\
\hline Dépression & 296.2, 296.3, 298.0, 300.4, 311 & F32 à F34 & 2 & $\geq 1 \mathrm{H}$ ou $\geq 4 \mathrm{M}$ \\
\hline Anxiété & $300.0,300.2$ & $\mathrm{~F} 40, \mathrm{~F} 41$ & 2 & $\geq 1 \mathrm{H}$ ou $\geq 3 \mathrm{P}$ \\
\hline
\end{tabular}

Abréviations : CIM, Classification internationale des maladies; H, demande de remboursement d'hôpitaux; M, demande de remboursement de médecins.

${ }^{\text {a }}$ Pour les demandes de remboursement de médecins, les codes de la CIM-9 ont été tronqués après le troisième chiffre.

deux sources de données. Les 604 patients avaient été sélectionnés à partir de deux études $^{26,27}$. Ils avaient eux-mêmes fourni au moyen d'un questionnaire ${ }^{27}$ la liste des affections concomitantes dont ils souffraient, ils avaient accepté qu'on examine leur dossier médical et ils avaient consenti au couplage de leurs données administratives et cliniques. Dans chacune des deux études, un responsable de l'extraction des données dûment formé et n'ayant pas accès aux données administratives avait utilisé le formulaire standardisé de collecte de données pour extraire les données sur les affections concomitantes à partir du dossier médical. On a posé aux participants des questions portant sur une liste plus longue d'affections concomitantes dans l'une des études, mais la présentation des questions était la même dans les deux études. Les résultats concernant la validité des définitions administratives de cas par rapport à l'examen des dossiers médicaux ont été publiés ailleurs $^{17-20}$.

Pour cette étude, nous avons choisi d'évaluer de manière plus approfondie certaines définitions de cas présentant des caractéristiques de performance raisonnables ${ }^{17-19,23,28}$ par rapport aux dossiers médicaux sur le plan de la sensibilité, de la spécificité et de la valeur kappa. Le tableau 1 indique les codes de CIM- 9 et 10 et la combinaison du nombre de demandes de remboursement par les hôpitaux et les médecins et du nombre d'années de données nécessaire pour satisfaire à ces diverses définitions de cas.
Comme il est possible que la sensibilité et la spécificité des données liées aux autodéclarations et des données liées aux dossiers médicaux en ce qui concerne les affections concomitantes diffèrent et comme le fait d'utiliser des normes de référence différentes est susceptible d'entraîner une hétérogénéité superflue au moment de comparer la validité des définitions de cas administratives entre les études ${ }^{29}$, nous avons mesuré la performance des définitions de cas administratives par rapport aux autodéclarations, de manière à faciliter les comparaisons avec la Nouvelle-Écosse, où la norme de référence était l'autodéclaration.

Les personnes répondant aux définitions de cas étaient considérées comme atteintes d'une affection à partir de la date de la première demande de remboursement de service de santé en lien avec cette affection.

\section{Nouvelle-Écosse}

\section{Contexte}

La Nouvelle-Écosse compte environ 945000 habitants $^{30}$. Les sources de données pour l'étude étaient les données (administratives) sur les demandes de remboursement de services de santé provinciales pour la Nouvelle-Écosse détenues par l'Unité de recherche sur la santé de la population de l'Université Dalhousie pour 1990-2010 ainsi que les autodéclarations provenant des patients de la Dalhousie Multiple Sclerosis Research Unit (DMSRU). Le Comité d'éthique de la recherche de la Régie de santé de la capitale de la Nouvelle-
Écosse a donné son approbation pour cette portion de l'étude.

\section{Données administratives}

Le programme public d'assurance maladie de la Nouvelle-Écosse finance les soins de tous les résidants et possède des dossiers informatisés de toutes les demandes de remboursement de services de santé, en particulier celles faites par les hôpitaux, les médecins et les pharmaciens. Chaque résidant a un numéro d'identification unique pour ses soins de santé, numéro qui figure dans chaque demande de remboursement de services de santé. Le registre des patients assurés fournit le sexe, la date de naissance et la date de décès de chaque bénéficiaire de services de santé ainsi que la période de validité de la protection offerte par l'assurance maladie de la province.

Dans la base de données sur les congés des hôpitaux sont notés pour chaque patient son numéro d'identification pour soins de santé et les diagnostics qu'il a reçus, ces derniers étant consignés au moyen d'un code à 5 chiffres de la CIM-9 (jusqu'en 2001) ou d'un code de la CIM-10-CA (depuis 2001). Nous nous sommes servis de tous les diagnostics consignés dans la base de données sur les congés des patients. On pouvait inscrire jusqu'à 5 diagnostics entre 1989 et 1991, 7 entre 1992 et 1995 et on peut en inscrire jusqu'à 16 depuis 1996.

Les demandes de remboursement soumises par les médecins incluent le numéro 
d'identification pour soins de santé du patient, la date du service et un code de la CIM-9 pour le diagnostic. Les codes de la CIM-9 étaient formés de 3 chiffres avant 1997 et on peut depuis 1997 inscrire jusqu'à 5 chiffres, mais les codes à 3 chiffres demeurent les plus utilisés ${ }^{21}$. Nous nous sommes servis des codes de la CIM-9 à 3 chiffres en tronquant des 2 derniers chiffres du code (tableau 1) dans le cas des demandes de remboursement faites par les médecins pour assurer la comparabilité avec les définitions de cas administratives élaborées au Manitoba, l'uniformité au fil du temps en Nouvelle-Écosse et la généralisation potentielle des résultats à d'autres provinces, notamment la Saskatchewan, l'Ontario, l'Île-du-Prince-Édouard et TerreNeuve-et-Labrador, qui utilisent également des codes à 3 chiffres $^{21}$. Ce choix concorde également avec la méthode utilisée par le Système national de surveillance des maladies chroniques (SNSMC, anciennement Système national de surveillance du diabète) : le SNSMC a élaboré des définitions de cas pour des maladies chroniques comme le diabète en se fondant sur le " plus petit dénominateur commun » afin de pouvoir appliquer ces définitions à toutes les provinces ${ }^{16}$. Les médecins de la Nouvelle-Écosse inscrivaient un seul diagnostic par demande de remboursement entre 1989 et 1996, alors qu'ils peuvent en soumettre jusqu'à 3 depuis 1997. Là aussi, dans le but d'assurer la comparabilité et la généralisation potentielle de ces définitions pour l'ensemble du Canada et au fil du temps, nous n'avons utilisé pour notre analyse primaire que le diagnostic primaire (le premier) inscrit par le médecin dans la demande de remboursement. Nous avons en revanche évalué l'impact de l'utilisation des 3 diagnostics inscrits dans les demandes des médecins au moment de l'analyse de sensibilité, dans le but d'améliorer la sensibilité des données administratives.

\section{Population de la Nouvelle-Écosse à l'étude}

Après avoir élaboré et testé plusieurs définitions administratives de cas pour la SP en les comparant aux données des dossiers médicaux (incluant lettres cliniques, rapports d'IRM, résultats d'analyse de liquide céphalorachidien et rapports sur les potentiels évoqués ${ }^{26}$ ), nous avons évalué leurs performances en Nouvelle-
Écosse $^{31}$. Nous avons à la suite de ce travail choisi d'appliquer la définition de cas consistant en au moins 3 demandes de remboursement par un hôpital (quel que soit le champ diagnostique) ou un médecin (diagnostic primaire seulement) concernant la SP. Nous avons appliqué à cette population les définitions de cas des affections concomitantes élaborées au Manitoba, à l'exception de celle de la maladie inflammatoire chronique de l'intestin, car le petit nombre de cas signalés avait empêché la validation de cette dernière au sein la population du Manitoba atteinte de SP. Nous avons utilisé à la place une définition de cas validée à l'échelle des populations générales du Manitoba ${ }^{32}$ et du Québec ${ }^{33}$.

\section{Base de données cliniques de la Dalhousie Multiple Sclerosis Research Unit}

La DMSRU, hébergée dans un hôpital de soins tertiaires, est le seul fournisseur de soins spécialisés dans le domaine de la SP en Nouvelle-Écosse. Chaque consultation par un patient y est consignée depuis 1980. Depuis 1998, tous les résidants de la Nouvelle-Écosse désirant bénéficier des fonds provinciaux pour un traitement lié à la SP (modifiant l'évolution de la maladie) se soumettent à une évaluation annuelle à la DMSRU ${ }^{34}$. Les patients qui fréquentent la DMSRU consentent à ce que leurs données cliniques soient couplées avec les données administratives à des fins de recherche. Au 31 décembre 2010, la base de données de la DMSRU contenait des données sur 4409 patients évalués ou traités pour une SP et le suivi clinique moyen associé était de 5,37 ans (écart-type [ÉT] : 6,46). Parmi eux, 2751 étaient atteints d'une SP confirmée et la durée moyenne de leur suivi clinique était de 7,78 ans (ÉT : 6,64).

Cohorte de validation de la Nouvelle-Écosse La DMSRU a commencé à inscrire les affections concomitantes lors de la première consultation clinique à partir de septembre 2006 et elle le fait depuis annuellement, au moyen d'un questionnaire uniformisé rempli par le patient. On considère comme présente une affection concomitante si le patient la déclare. On demande au patient de déclarer toutes les affections concomitantes dont il souffre, indépendamment de la date de diagnostic, de manière à prendre en compte celles qui sont asymptomatiques au moment de la consultation (par exemple les migraines en rémission). Au 31 décembre 2010, la cohorte de la DMSRU était constituée de 1923 patients atteints de SP confirmée, dont la durée moyenne de suivi clinique était de 8,83 ans (ÉT : 6,60) et dont l'âge moyen d'apparition des symptômes de SP était de 33,2 ans (ÉT : 9,9) (tableau 2). À l'aide des numéros uniques d'identification pour soins de santé, nous avons couplé cette cohorte de validation aux données administratives concernant la population de Nouvelle-Écosse à l'étude atteinte de SP pour la période comprise entre le $1^{\mathrm{er}}$ janvier 1990 (date la plus ancienne pour laquelle nous avons accès aux données administratives) et le 31 décembre 2010.

\section{Analyses statistiques}

Nous avons d'abord comparé, pour les cohortes de validation, la classification des affections concomitantes provenant des définitions administratives de cas et celle provenant des diagnostics. Nous avons calculé ensuite pour chaque affection concomitante la sensibilité et la spécificité de la définition administrative de cas par rapport aux autodéclarations avec des intervalles de confiance à $95 \%$ fondés sur une distribution binomiale. Étant donné que ni les données administratives ni les autodéclarations ne pouvaient être considérées comme la référence idéale ou privilégiée des données sur les affections concomitantes ${ }^{14,35}$, nous avons estimé la concordance entre les sources de données à l'aide de la valeur kappa $(\kappa)$, méthode utilisée justement lorsqu'aucune des sources de données ne peut être considérée comme la norme de référence. La valeur kappa indique le degré de concordance au-delà de ce qui est imputable au hasard et elle est calculée comme suit : (concordance observée - concordance due au hasard $) \div(1$ - concordance due au hasard). Nous avons interprété les valeurs $\kappa$ obtenues comme suit : concordance faible $(0$ à 0,20$)$, médiocre $(0,21$ à $0,40)$, modérée $(0,41$ à 0,60$)$, bonne $(0,61$ à 0,80$)$ et presque parfaite $(0,81 \text { à } 1,0)^{36}$. Les cohortes de validation les plus réduites au début de l'étude comportant 606 individus, nous nous attendions à ce qu'elles nous permettent de détecter une 
TABLEAU 2

Caractéristiques des cohortes de validation utilisées pour identifier les affections concomitantes au sein de la population atteinte de sclérose en plaques, Nouvelle-Écosse et Manitoba (Canada)

\begin{tabular}{|c|c|c|c|c|}
\hline Caractéristiques & \multicolumn{2}{|c|}{ Cohorte de validation du Manitoba } & \multicolumn{2}{|c|}{ Cohorte de validation de la Nouvelle-Écosse } \\
\hline Effectifs $(\mathrm{N})$ & \multicolumn{2}{|c|}{606} & \multicolumn{2}{|c|}{1923} \\
\hline Période visée par les autodéclarations des participants & \multicolumn{2}{|c|}{ Toute la vie jusqu'en 2006} & \multicolumn{2}{|c|}{$\begin{array}{l}\text { Toute la vie jusqu'au début de la collecte des } \\
\text { données, soit de septembre } 2006 \text { à décembre } 2010\end{array}$} \\
\hline $\begin{array}{l}\text { Période visée par les données administratives (demandes de } \\
\text { remboursement d'hôpitaux et de médecins) }\end{array}$ & \multicolumn{2}{|c|}{$1^{\mathrm{er}}$ avril 1984 au 31 mars 2007} & \multicolumn{2}{|c|}{$1^{\text {er }}$ janvier 1990 au 31 décembre 2010} \\
\hline Nombre de femmes (\%) & \multicolumn{2}{|c|}{$511(84,4)$} & \multicolumn{2}{|c|}{$1480(77,0)$} \\
\hline Source des données sur les affections concomitantes & Autodéclarations & Données administratives & Autodéclarations & Données administratives \\
\hline Hypertension (\%) & $91 / 576(15,8)$ & $89 / 576(15,4)$ & $466(24,2)$ & $500(26,0)$ \\
\hline Hyperlipidémie (\%) & $10 / 169(5,9)$ & $12 / 169(7,1)$ & $329(17,1)$ & $257(13,3)$ \\
\hline Diabète (\%) & $30 / 606(4,9)$ & 29/606 (4,8) & $127(6,6)$ & $151(7,8)$ \\
\hline Dépression (\%) & $31 / 128(24,2)$ & $23 / 128(18,0)$ & $562(29,2)$ & $522(27,1)$ \\
\hline Anxiété (\%) & $58 / 405(14,3)$ & $41 / 405(10,1)$ & $339(17,6)$ & $614(31,9)$ \\
\hline
\end{tabular}

Abréviation : ÉT, écart-type.

- : Donnée non disponible.

valeur $\kappa$ supérieure ou égale à 0,60 si les affections concomitantes affectaient $3 \%$ ou plus de la cohorte, et notre hypothèse nulle était une valeur $\kappa$ supérieure ou égale à 0,40 (plus faible valeur $\kappa$ acceptable), avec $\alpha=0,05$ et $\beta=0,20$.

Les analyses statistiques ont été effectuées à l'aide du logiciel SAS version 9.1 (SAS Institute Inc., Cary, Caroline du Nord, États-Unis).

\section{Résultats}

Le tableau 2 détaille les caractéristiques des cohortes de validation du Manitoba et de la Nouvelle-Écosse. Les distributions en fonction de l'âge et du sexe correspondent pour les deux cohortes aux résultats attendus pour une population atteinte de $\mathrm{SP}^{26}$. Les fréquences établies à partir des réponses autodéclarées sur la dépression, l'anxiété, le diabète et la cardiopathie sont semblables dans les deux cohortes de validation, alors que l'hypertension et l'hyperlipidémie semblent moins fréquentes au Manitoba qu'en NouvelleÉcosse. Dans les deux provinces, et pour chaque affection concomitante, les fréquences obtenues à l'aide des données administratives et celles obtenues à partir des autodéclarations des participants sont généralement semblables (tableau 2).

La spécificité de la définition administrative de cas par rapport aux autodéclarations a dépassé $93 \%$ au Manitoba pour toutes les affections (tableau 3). Les résultats ont été similaires en Nouvelle-Écosse, avec une spécificité de toutes les définitions de cas dépassant $91 \%$, sauf pour la dépression (82\%) et l'anxiété (74\%). Dans les deux provinces, la sensibilité des définitions administratives de cas a varié davantage que la spécificité. La sensibilité a été particulièrement faible pour l'anxiété, descendant jusqu’à $29 \%$ au Manitoba.

Au Manitoba, la concordance entre les définitions administratives de cas et les autodéclarations a été bonne pour l'hypertension et le diabète, modérée pour l'hyperlipidémie, la cardiopathie et la dépression et médiocre pour l'anxiété. En NouvelleÉcosse, on a observé une concordance légèrement moins bonne pour l'hypertension et l'hyperlipidémie et semblable pour le diabète et la cardiopathie. Pour la dépression, la concordance y a été inférieure et plus divergente. La spécificité de la définition de cas pour l'anxiété a été plus faible en Nouvelle-Écosse qu'au Manitoba. Deux affections n'ont été analysées qu'en Nouvelle-Écosse : la maladie inflammatoire chronique de l'intestin, pour laquelle la concordance s'est révélée bonne, et l'épilepsie, pour laquelle elle s'est révélée modérée.

Dans le cadre de l'analyse de sensibilité, nous avons également évalué l'effet de l'utilisation des 3 diagnostics inscrits sur les demandes de remboursement de médecins plutôt que du premier seulement, ce qui n'a pas eu d'effet notoire sur la performance des définitions de cas (voir tableau 3).

\section{Analyse}

Nous avons analysé dans deux provinces canadiennes, la Nouvelle-Écosse et le Manitoba, la performance des définitions administratives de cas pour les affections concomitantes touchant couramment la population atteinte de SP. La prévalence de ces affections concomitantes différait quelque peu entre les deux populations, ce qui cadre avec la variation observée dans d'autres études ${ }^{11}$. Notre étude a révélé que plusieurs définitions d'affections concomitantes importantes établies à partir des 
TABLEAU 3

Performance des définitions des affections concomitantes utilisées dans les demandes de remboursement par rapport aux autodéclarations des sujets atteints de sclérose en plaques, Nouvelle-Écosse et Manitoba (Canada)

\begin{tabular}{|c|c|c|c|c|c|c|}
\hline Affection concomitante & $\begin{array}{l}\text { Province (nombre de } \\
\text { champs diagnostiques) }\end{array}$ & $\begin{array}{l}\text { Sensibilité } \\
\text { (IC à } 95 \%)\end{array}$ & $\begin{array}{l}\text { Spécificité } \\
\text { (IC à } 95 \%)\end{array}$ & VPP (IC à 95 \%) & VPN (IC à 95 \%) & Kappa (IC à 95 \%) \\
\hline \multirow[t]{3}{*}{ Hypertension } & Manitoba (1) & $0,80(0,71$ à 0,88$)$ & $0,96(0,94$ à 0,98$)$ & $0,79(0,69$ à 0,87$)$ & $0,96(0,94$ à 0,98$)$ & $0,76(0,69$ à 0,84$)$ \\
\hline & Nouvelle-Écosse (1) & $0,79(0,75$ à 0,83$)$ & $0,91(0,89$ à 0,92$)$ & $0,74(0,70$ à 0,77$)$ & $0,93(0,92$ à 0,94$)$ & $0,68(0,64$ à 0,72$)$ \\
\hline & Nouvelle-Écosse (3) & $0,79(0,75$ à 0,83$)$ & $0,90(0,89$ à 0,92$)$ & $0,73(0,68$ à 0,76$)$ & $0,93(0,92$ à 0,94$)$ & $0,68(0,64$ à 0,71$)$ \\
\hline \multirow[t]{3}{*}{ Hyperlipidémie } & Manitoba (1) & $0,50(0,21$ à 0,97$)$ & $0,97(0,94$ à 0,99$)$ & $0,60(0,26$ à 0,88$)$ & $0,96(0,92$ à 0,99$)$ & $0,51(0,25$ à 0,78$)$ \\
\hline & Nouvelle-Écosse (1) & $0,53(0,48$ à 0,59$)$ & $0,95(0,94$ à 0,96$)$ & $0,68(0,62$ à 0,74$)$ & $0,91(0,89$ à 0,92$)$ & $0,53(0,47$ à 0,58$)$ \\
\hline & Nouvelle-Écosse (3) & $0,56(0,50$ à 0,61$)$ & $0,94(0,93$ à 0,95$)$ & $0,67(0,61$ à 0,73$)$ & $0,91(0,90$ à 0,93$)$ & $0,54(0,49$ à 0,59$)$ \\
\hline \multirow[t]{3}{*}{ Diabète } & Manitoba (1) & $0,67(0,47$ à 0,83$)$ & 0,98 (0,97 à 0,99) & $0,60(0,36$ à 0,81$)$ & $0,99(0,98$ à 0,99$)$ & $0,66(0,52$ à 0,80$)$ \\
\hline & Nouvelle-Écosse (1) & $0,80(0,71$ à 0,86$)$ & 0,97 (0,96 à 0,98) & $0,67(0,59$ à 0,74$)$ & $0,99(0,98$ à 0,99$)$ & $0,70(0,64$ à 0,77$)$ \\
\hline & Nouvelle-Écosse (3) & $0,80(0,71$ à 0,86$)$ & 0,97 (0,96 à 0,98) & $0,67(0,58$ à 0,74$)$ & $0,99(0,98$ à 0,99$)$ & $0,70(0,64$ à 0,77$)$ \\
\hline \multirow[t]{3}{*}{ Cardiopathie } & Manitoba (1) & $0,42(0,23$ à 0,63$)$ & $0,99(0,98$ à 0,99$)$ & $0,69(0,41$ à 0,89$)$ & $0,97(0,96$ à 0,98$)$ & $0,51(0,32$ à 0,69$)$ \\
\hline & Nouvelle-Écosse (1) & $0,58(0,46$ à 0,71$)$ & $0,96(0,95$ à 0,97$)$ & $0,36(0,27$ à 0,46$)$ & $0,99(0,98$ à 0,99$)$ & $0,42(0,33$ à 0,52$)$ \\
\hline & Nouvelle-Écosse (3) & $0,58(0,46$ à 0,71$)$ & $0,96(0,95$ à 0,97$)$ & $0,36(0,27$ à 0,46$)$ & $0,99(0,98$ à 0,99$)$ & $0,42(0,33$ à 0,52$)$ \\
\hline \multirow{2}{*}{$\begin{array}{l}\text { Maladie inflammatoire } \\
\text { chronique de l'intestin }{ }^{a}\end{array}$} & Nouvelle-Écosse (1) & $0,59(0,41$ à 0,76$)$ & $1,0(1,0$ à 1,0$)$ & $0,90(0,70$ à 0,99$)$ & $0,99(0,99$ à 1,0$)$ & $0,71(0,57$ à 0,85$)$ \\
\hline & Nouvelle-Écosse (3) & $0,59(0,41$ à 0,76$)$ & $1,0(1,0$ à 1,0$)$ & $0,90(0,70$ à 0,99$)$ & $0,99(0,99$ à 1,0$)$ & $0,71(0,57$ à 0,85$)$ \\
\hline \multirow[t]{2}{*}{ Épilepsie ${ }^{a}$} & Nouvelle-Écosse (1) & $0,47(0,34$ à 0,61$)$ & $0,99(0,98$ à 0,99$)$ & $0,51(0,37$ à 0,65$)$ & 0,98 (0,98 à 0,99) & $0,48(0,36$ à 0,60$)$ \\
\hline & Nouvelle-Écosse (3) & $0,47(0,34$ à 0,61$)$ & $0,99(0,98$ à 0,99$)$ & $0,51(0,37$ à 0,65$)$ & $0,98(0,98$ à 0,99$)$ & $0,48(0,36$ à 0,60$)$ \\
\hline \multirow[t]{3}{*}{ Dépression } & Manitoba (1) & $0,55(0,36$ à 0,73$)$ & $0,94(0,87$ à 0,98$)$ & $0,74(0,52$ à 0,90$)$ & $0,87(0,79$ à 0,92$)$ & $0,53(0,36$ à 0,71$)$ \\
\hline & Nouvelle-Écosse (1) & $0,49(0,45$ à 0,54$)$ & $0,82(0,80$ à 0,84$)$ & $0,53(0,48$ à 0,57$)$ & $0,80(0,77$ à 0,82$)$ & $0,32(0,27$ à 0,36$)$ \\
\hline & Nouvelle-Écosse (3) & $0,49(0,45$ à 0,54$)$ & $0,82(0,80$ à 0,84$)$ & $0,53(0,48$ à 0,57$)$ & $0,80(0,77$ à 0,82$)$ & $0,32(0,27$ à 0,36$)$ \\
\hline \multirow[t]{3}{*}{ Anxiété } & Manitoba (1) & $0,29(0,18$ à 0,43$)$ & $0,93(0,90$ à 0,95$)$ & $0,41(0,26$ à 0,58$)$ & $0,89(0,85$ à 0,92$)$ & $0,26(0,13$ à 0,38$)$ \\
\hline & Nouvelle-Écosse (1) & $0,62(0,56$ à 0,67$)$ & $0,74(0,72$ à 0,77$)$ & $0,34(0,30$ à 0,38$)$ & $0,90(0,88$ à 0,92$)$ & $0,27(0,23$ à 0,32$)$ \\
\hline & Nouvelle-Écosse (3) & $0,62(0,57$ à 0,67$)$ & $0,74(0,72$ à 0,76$)$ & $0,34(0,30$ à 0,38$)$ & $0,90(0,88$ à 0,92$)$ & $0,27(0,23$ à 0,32$)$ \\
\hline
\end{tabular}

Abréviations : IC, intervalle de confiance; VPN, valeur prédictive négative; VPP, valeur prédictive positive.

${ }^{\text {a }}$ Nombre insuffisant de cas signalés au Manitoba $(<5)$.

données administratives sur la santé des populations atteintes de MS dans ces deux provinces offraient de bonnes performances générales. Il s'agit d'un pas majeur dans l'élaboration d'un système de surveillance pancanadien des affections concomitantes de la SP, enjeu d'importance croissante pour la population, le système de santé et les décideurs.

Notre étude a fait en outre ressortir l'importance d'élaborer une méthodologie robuste et de bien comprendre les points forts et les limites des sources de données disponibles avant de procéder à la mise en place de ce système. Par exemple, on a constaté que les définitions administratives de cas ne présentaient pas le même niveau de concordance pour toutes les affections concomitantes.

Les résultats obtenus montrent également qu'il est important que la performance des définitions de cas élaborées à l'intérieur d'une province ou d'une population soit mesurée avant d'appliquer ces définitions ailleurs.

Les données administratives ont l'avantage d'être faciles d'accès, peu onéreuses et fondées sur la population. Toutefois, étant donné qu'elles sont recueillies pour la gestion des systèmes de santé, elles doivent être validées avant d'être utilisées pour une recherche. Leur utilité est variable selon la population et l'affection étudiées $^{25,29}$, et leur validité semble différer en fonction du plan d'étude ${ }^{29}$. Alors que la sensibilité de nos définitions administratives de cas s'est révélée variable, leur spécificité est demeurée élevée, dépassant généralement $90 \%$ pour les affections concomitantes physiques, et ce, conformément à nos observations antérieures au Manitoba ${ }^{17-19,23}$. La constance de la performance des définitions de cas pour la population atteinte de SP au Manitoba et en Nouvelle-Écosse justifie l'utilisation de celles-ci dans d'autres provinces canadiennes. Alors que la spécificité pour les affections concomitantes physiques était élevée, celle concernant les affections concomitantes psychiatriques était légèrement plus faible, variant entre $74 \%$ et $94 \%$. Dans d'autres études de validation des définitions administratives de cas au Canada, on a noté qu'il était difficile de distinguer la dépression de l'anxiété à l'aide des codes à trois chiffres de la CIM étant donné leur manque de spécificité à ce niveau ${ }^{37,38}$. Par exemple, un même code (300) décrit le trouble dysthymique (300.4), qui est une forme de dépression chronique, et les états anxieux (300.0). De même, le code 296 est utilisé pour décrire à la fois un épisode dépressif majeur isolé (296.2) et un épisode manique isolé du trouble bipolaire (296.0). Outre cette difficulté à distinguer dépres- 
sion et anxiété, on a relevé, lors d'autres tentatives de validation des définitions de cas pour la dépression, une mauvaise concordance avec la mesure de la dépression de la Composite International Diagnostic Interview ${ }^{38}$. Une étude portant sur une population traitée par antidépresseurs en Saskatchewan (Canada) a révélé que la concordance entre la dépression définie à partir des demandes de remboursement des médecins et la dépression inscrite dans les dossiers médicaux était modérée $(\kappa=0,54)$, un résultat supérieur à ce que nous avons observé ${ }^{39}$. Au Manitoba, où l'on dispose de données sur les demandes de remboursement d'ordonnances pour la population, nous avons constaté que les définitions administratives de cas qui fonctionnaient le mieux pour les affections concomitantes psychiatriques, dont la dépression et l'anxiété, étaient celles qui étaient fondées sur ces demandes de remboursement d'ordonnances ${ }^{18}$. Comme la Nouvelle-Écosse ne dispose cependant pas, quant à elle, de bases de données sur les ordonnances pour sa population - ce qui est également le cas de plusieurs autres provinces canadiennes -, cela limite l'utilisation à l'échelle nationale des définitions fondées sur les demandes de remboursement d'ordonnances. De plus, la concordance pour la dépression entre les définitions de cas et les autodéclarations était également plus faible en NouvelleÉcosse qu'au Manitoba. Il faut donc redoubler de prudence lorsqu'on utilise des définitions de cas pour les affections concomitantes psychiatriques.

Plusieurs des définitions de cas que nous avons utilisées avaient été élaborées à l'origine pour la population générale, mais elles ont été considérées comme adéquates pour la population atteinte de SP lorsqu'on les a comparées à d'autres définitions de cas testées au Manitoba ${ }^{17,19,28}$. Parmi celles-ci, on retrouve les définitions de cas pour l'hypertension, la cardiopathie et la maladie inflammatoire chronique de l'intestin. Nous avons utilisé la définition de l'hypertension du SNSMC, ce qui a donné des résultats aussi performants dans les deux populations atteintes de SP à l'étude : nous avons observé une sensibilité variant entre $79 \%$ et $80 \%$, une spécificité variant entre $91 \%$ et $96 \%$ et une valeur $\kappa$ variant entre 0,68 et 0,76. Dans les études menées en population générale en Ontario et au Manitoba où ces définitions de cas ont été mises à l'épreuve, on a relevé une sensibilité variant entre $69 \%$ et $73 \%$, une spécificité de $95 \%$ et une valeur $\kappa$ variant entre 0,67 et $0,70^{25,40}$. Quant à la cardiopathie, sa définition de cas a offert une concordance modérée avec les autodéclarations au Manitoba $(\kappa=0,51)$ et en Nouvelle-Écosse $(\kappa=0,42)$, ce qui cadre avec les résultats obtenus au Manitoba pour la population générale $(\kappa=0,55)^{25}$. En ce qui concerne la maladie inflammatoire chronique de l'intestin, nous avons appliqué à la population atteinte de SP de la Nouvelle-Écosse une définition de cas élaborée pour la population générale du Manitoba, et nous avons obtenu une sensibilité de $59 \%$, une spécificité de $100 \%$ et une valeur $\kappa$ de 0,71. La spécificité et le degré de concordance se comparent favorablement aux résultats initiaux obtenus au Manitoba lors de l'utilisation des autodéclarations (spécificité de $91 \%$ et valeur $\kappa$ variant entre 0,79 et 0,80 ), mais la sensibilité est plus faible que celle relevée pour la population générale au Manitoba ( $>87 \%$ ) ou au Québec (97 \%). Nous avons également obtenu une sensibilité plus faible lorsque nous avons appliqué à la population atteinte de SP des définitions de cas du diabète et de l'épilepsie qui avaient été conçues pour la population générale. Nous avions évalué au préalable la performance de la définition du diabète du SNSMC dans la population atteinte de SP au Manitoba, et nous avions alors obtenu une sensibilité de seulement $50 \%$, un chiffre considérablement inférieur aux taux variant entre $79,5 \%$ et $86 \%$ pour la population générale $e^{25,41}$. Nous avons découvert qu'en utilisant 5 années de données plutôt que 2 pour la définition de cas du SNSMC, la sensibilité s'améliorait ${ }^{17}$ : elle passait à $80 \%$ pour la population de la Nouvelle-Écosse, tout en conservant une spécificité élevée (97\%). En ce qui concerne l'épilepsie, nous avons relevé une sensibilité de $47 \%$ et une spécificité de $99 \%$ par rapport aux autodéclarations. La définition que nous avons employée pour l'épilepsie - 1 hospitalisation ou 2 demandes de remboursement par un médecin en 3 ans - n’a pas été testée pour la population générale du Canada, mais une définition de cas reposant sur 1 hospitalisation ou 2 demandes de remboursement de médecins en 2 ans a été associée, pour la population générale de l'Alberta, à une sensibilité considérablement supérieure $(88,9 \%)$ pour une spécificité similaire $(92,4 \%)^{42}$. Globalement, si l'on en croit ces résultats, les définitions de cas élaborées pour une population générale pourraient ne pas être aussi performantes lorsqu'on les applique à des populations atteintes de certaines maladies chroniques, ce qui signifie qu'on doit évaluer leur validité avant de les utiliser.

Une sensibilité des définitions de cas pour les maladies concomitantes plus faible lors de l'application à la population atteinte de SP que lors de l'application à la population générale pourrait relever des limites connues des données administratives : les affections concomitantes seraient sousdéclarées dans les demandes de remboursement d'hôpitaux en raison de biais de codage $^{43}$. La sensibilité des définitions de cas pourrait également être influencée par le nombre de codes diagnostiques inscrits, en particulier dans les demandes de remboursement effectuées par les médecins, étant donné que les soins pour les maladies chroniques sont fournis la plupart du temps en consultation externe. Le nombre de champs diagnostiques remplis dans les demandes de remboursement de médecins varie, allant de 1 seulement dans la plupart des provinces à 3 en Colombie-Britannique, en Alberta ou en Nouvelle-Écosse (depuis 1997), 2 au Yukon avant 2006 (nombre illimité depuis) et 11 au Nunavut ${ }^{21}$. C'est pour cette raison que nous avons évalué l'effet qu'aurait l'utilisation de 3 diagnostics plutôt que d'un seul. Bien qu'aucune influence majeure du nombre de champs diagnostiques utilisés sur la sensibilité ou la spécificité de nos définitions de cas n’ait été relevée, il faudrait faire ces vérifications dans les autres provinces ou territoires où 3 champs diagnostiques sont disponibles.

Nous n'avons pas tenté d'évaluer l'effet des changements dans la facturation à l'acte lorsque les médecins ont changé de régime de rémunération, mais ce facteur est également susceptible de varier d'une province à l'autre ${ }^{21}$ et d'influer sur la sensibilité des données administratives. 
Nous avons utilisé les autodéclarations comme norme de référence, tout en sachant qu'aucune source de données ne peut être considérée comme la norme idéale pour les affections concomitantes, et que les cas de discordance entre les données provenant des autodéclarations et les données administratives pourraient être attribuables aux limites inhérentes à ces deux sources de données. Collecter des données robustes provenant d'autodéclarations comporte en effet des difficultés inhérentes à l'autodéclaration : les répondants peuvent ne pas se rappeler toutes les affections médicales qu'on leur a diagnostiquées, ne pas signaler certaines affections à cause d'un biais de désirabilité sociale ou encore ne pas avoir bien compris les diagnostics qu'ils ont reçus. Il demeure néanmoins souvent plus facile et plus rentable de recueillir ces données plutôt que d'accéder à l'ensemble de l'information contenue dans les dossiers médicaux. On a par ailleurs constaté qu'en examinant les dossiers provenant d'un seul fournisseur de soins, on obtenait une sensibilité pour les maladies chroniques plus faible qu'à l'examen des dossiers de tous les fournisseurs qu'une personne avait consultés ${ }^{44}$, mais il est clair que l'accès à l'ensemble des dossiers peut être complexe, que ce soit pour des raisons de coût, de confidentialité ou d'autres raisons d'ordre pratique. Par ailleurs, un indice de comorbidité établi à partir de données administratives a permis d'obtenir des résultats supérieurs à ceux obtenus avec un indice de comorbidité fondé sur l'examen des données provenant des dossiers hospitaliers d'un seul jour ${ }^{45}$. Bien que la validité des données provenant des autodéclarations se soit révélée variable en fonction de l'affection considérée, ces données semblent raisonnablement exactes dans le cas de troubles chroniques bien définis exigeant des soins continus ou causant une incapacité, ce qui est le cas de ceux faisant l'objet de notre étude $^{14}$. En outre, les autodéclarations sont susceptibles de prédire la qualité de vie liée à la santé et l'état fonctionnel avec plus de précision que les données tirées des dossiers médicaux ${ }^{46}$. Pris ensemble, ces résultats indiquent que, pour certaines affections chroniques du moins, les données provenant des autodéclarations fournissent un outil adéquat de comparaison lors de la validation des définitions administratives de cas.

Parmi les points forts de notre étude, notons la large taille des cohortes utilisées et la disponibilité en données comparables au Manitoba et en Nouvelle-Écosse. De plus, nous avons constaté que les caractéristiques de performance des définitions administratives de cas pour plusieurs affections concomitantes couramment observées en lien avec la SP étaient relativement stables d'une province à l'autre, ce qui fait que ces définitions pourraient vraisemblablement être efficaces dans des études populationnelles portant sur l'épidémiologie et l'effet des affections concomitantes de la SP. Il reste à optimiser les définitions de cas des affections concomitantes psychiatriques et à entreprendre d'autres travaux pour élaborer des méthodes valides pour l'évaluation d'autres affections concomitantes potentiellement importantes au sein de la population atteinte de SP.

\section{Mentions de conflit d'intérêt et remerciements}

Cette étude a été partiellement financée par des subventions Don Paty Career Development à Ruth Ann Marrie provenant de la Société canadienne de la sclérose en plaques, des IRSC et de la Fondation pour la recherche en santé des Compagnies de recherche pharmaceutique du Canada. Ces sources de financement n'ont joué aucun rôle dans le plan d'études, dans la collecte, l'analyse ou l'interprétation des données, ou dans la décision de soumettre l'article pour publication. Les résultats et conclusions présentés sont ceux des auteurs. N'y ont souscrit ni Santé Manitoba ni l'Unité de recherche sur la santé de la population (Université Dalhousie), et on ne devrait donc pas leur attribuer.

Ruth Ann Marrie reçoit des fonds de recherche des IRSC, de l'Agence de la santé publique du Canada, du Conseil manitobain de la recherche en matière de santé, de la Health Sciences Centre Foundation, de la Société canadienne de la sclérose en plaques et de la Fondation pour la recherche en santé des Compagnies de recherche pharmaceutique et elle a mené des essais cliniques financés par Bayer et Sanofi-Aventis.
John Fisk est directeur du endMS Atlantic Regional Research and Training Centre, organisme financé par la Société canadienne de la sclérose en plaques. Il reçoit des fonds de recherche des IRSC et il a reçu des subventions, des honoraires et des honoraires d'expert-conseil d'AstraZeneca, de Bayer, de Biogen Idec Canada, de Heron Evidence Development Limited, de Hoffmann-La Roche, du MAPI Research Trust, de Novartis, de Sanofi-Aventis, de Serono Canada et de QualityMetric Incorporated.

Karen Stadnyk n'a aucun conflit d'intérêts à déclarer.

Helen Tremlett reçoit actuellement des fonds de la Société canadienne de la sclérose en plaques (Bourse Don Paty Career Development), de la National MS Society des É.-U. (n ${ }^{\circ}$ RG 4202-A-2 [CP]); des IRSC ( $n^{\text {os }}$ MOP 190898 [CP] et MOP93646 [CP]), de la Fondation Michael Smith pour la recherche en santé (Bourse de recherche) et du Programme de chaires de recherche du Canada. Elle a reçu des honoraires pour des conférences qu'elle a données ou elle a obtenu un remboursement de ses frais de déplacement pour sa participation à des conférences de la part du Consortium of MS Centers, de la National MS Society des É.-U., de la Swiss Multiple Sclerosis Society, du Multiple Sclerosis Research Program de l'Université de la Colombie-Britannique, de Teva Pharmaceutical Industries et de Bayer Pharmaceutical (honoraires refusés) et d'ECTRIMS. Sauf exception, les honoraires reçus en tant que conférencière ont été reversés à un organisme caritatif œuvrant dans le domaine de la SP ou ajoutés à une subvention non liée utilisée par son groupe de recherche.

Christina Wolfson reçoit des fonds de recherche de la Société canadienne de la sclérose en plaques, des IRSC, de la Fondation canadienne pour l'innovation, de la National Multiple Sclerosis Society des É.-U. et de l'Agence de la santé publique du Canada.

Sharon Warren reçoit des fonds de recherche des IRSC, de la Fondation canadienne de la recherche sur les ser- 
vices de santé, des Alberta Health Services et du CRSH.

Virender Bhan reçoit des fonds de recherche des IRSC et a agi à titre de conseiller ou expert-conseil pour le compte de Bayer, Biogen Idec, EMD Serono, Novartis et Teva Pharmaceutical Industries.

Nancy Yu reçoit des fonds de soutien de la recherche de l'Agence canadienne de développement international (ACDI), de la Société canadienne de la sclérose en plaques, des IRSC et de Santé et Vie saine Manitoba.

\section{Références}

1. Gijsen R, Hoeymans N, Schellevis FG, Ruwaard D, Satariano WA, van den Bos GA. Causes and consequences of comorbidity: a review. J Clin Epidemiol. 2001;54(7): 661-674.

2. Mielke MM, Rosenberg PB, Tschanz $\mathrm{J}$ et collab. Vascular factors predict rate of progression in Alzheimer disease. Neurology. 2007;69(19):1850-1858.

3. Dean G. How many people in the world have multiple sclerosis? Neuroepidemiology. 1994; 13(1-2):1-7.

4. Evans C, Beland S, Kulaga S et collab. Incidence and prevalence of multiple sclerosis in the Americas: a systematic review. Neuroepidemiology. 2013;40(3):195-210.

5. Warren SA, Svenson LW, Warren KG. Contribution of incidence to increasing prevalence of multiple sclerosis in Alberta, Canada. Mult Scler. 2008;14(7):872-879.

6. Beck CA, Metz LM, Svenson LW, Patten SB. Regional variation of multiple sclerosis prevalence in Canada. Mult Scler. 2005;11(5): 516-519.

7. Marrie RA, Cutter G, Tyry T. Substantial adverse association of visual and vascular comorbidities on visual disability in multiple sclerosis. Mult Scler. 2011;17(12):1464-1471.

8. Marrie RA, Horwitz RI, Cutter G, Tyry T, Campagnolo D, Vollmer T. Comorbidity delays diagnosis and increases disability at diagnosis in MS. Neurology. 2009;72(2): 117-124.
9. Marrie RA, Rudick R, Horwitz R et collab. Vascular comorbidity is associated with more rapid disability progression in multiple sclerosis. Neurology. 2010;74(13):10411047.

10. Warren SA, Turpin KV, Pohar SL, Jones CA, Warren KG. Comorbidity and health-related quality of life in people with multiple sclerosis. Int J MS Care. 2009;11(1):6-16.

11. Marrie RA, Horwitz RI. Emerging effects of comorbidities on multiple sclerosis. Lancet Neurol. 2010;9(8):820-828.

12. Institut canadien d'information sur la santé (ICIS). Le fardeau des maladies, troubles et traumatismes neurologiques au Canada. Ottawa (Ont.) : ICIS; 2007.

13. Lash TL, Mor V, Wieland D, Ferrucci L, Satariano W, Silliman RA. Methodology, design, and analytic techniques to address measurement of comorbid disease. J Gerontol A Biol Sci Med Sci. 2007;62(3): 281-285.

14. Okura Y, Urban LH, Mahoney DW, Jacobsen SJ, Rodeheffer RJ. Agreement between self-report questionnaires and medical record data was substantial for diabetes, hypertension, myocardial infarction and stroke but not for heart failure. J Clin Epidemiol. 2004;57(10):1096-2103.

15. Patten S. Integrating data from clinical and administrative databases in pharmacoepidemiological research. Can J Clin Pharmacol. 1998;5(2):92-97.

16. Santé Canada. Système national de surveillance du diabète. Relever le défi posé par le diabète au Canada - Premier rapport du Système national de surveillance du diabète (SNSD) 2003 [Internet]. Ottawa (Ont.) : Santé Canada; 2003 [consultation le 10 janv. 2006]. PDF (554 Ko) téléchargeable à partir du lien : http://publications.gc.ca /collections/Collection/H39-4-21-2003F.pdf

17. Marrie R, Yu B, Leung S et collab. Rising prevalence of vascular comorbidities in MS: validation of administrative definitions for diabetes, hypertension, hyperlipidemia. Mult Scler. 2012;18(9):1310-1319.

18. Marrie RA, Fisk JD, Yu BN et collab. Mental comorbidity and multiple sclerosis: validating administrative data to support population-based surveillance. BMC Neurol. 2013; 13(1):16.
19. Marrie RA, Yu BN, Leung $S$ et collab. The utility of administrative data for surveillance of comorbidity in multiple sclerosis: a validation study. Neuroepidemiology. 2012; 40(2):85-92.

20. Marrie RA, Yu BN, Leung $S$ et collab. The incidence and prevalence of thyroid disease do not differ in the multiple sclerosis and general populations: a validation study using administrative data. Neuroepidemiology. 2012;39(2):135-142.

21. Lix LM, Walker R, Quan H, Nesdole R, Yang J, Chen G. Features of physician services databases in Canada. Chronic Dis Inj Can. 2012;32(4):186-193.

22. Health Information Management Branch. Population report. Winnipeg (Man.) : Manitoba Health and Healthy Living; 2008 (1 $1^{\mathrm{er}}$ juin).

23. Marrie RA, Yu BN, Leung $S$ et collab. The incidence and prevalence of fibromyalgia are higher in multiple sclerosis than the general population: a population-based study. Mult Scler Rel Dis. 2012;1(4):162167.

24. Marrie RA, Horwitz R, Cutter G, Tyry T, Campagnolo D, Vollmer T. Comorbidity, socioeconomic status, and multiple sclerosis. Mult Scler. 2008;14(8):1091-1098.

25. Lix L, Yogendran M, Shaw S, Burchill C, Metge C, Bond R. Sources de données des populations utilisées en surveillance des maladies chroniques. Maladies chroniques au Canada. 2008;29(1):34-42.

26. Marrie RA, Yu N, Blanchard JF, Leung S, Elliott L. The rising prevalence and changing age distribution of multiple sclerosis in Manitoba. Neurology. 2010;74(6):465-471.

27. Horton M, Rudick RA, Hara-Cleaver C, Marrie RA. Validation of a self-report comorbidity questionnaire for multiple sclerosis. Neuroepidemiology. 2010;35(2):83-90.

28. Marrie RA, Yu BN, Leung $S$ et collab. Prevalence and incidence of ischemic heart disease in multiple sclerosis: a populationbased validation study. Mult Scler Rel Dis. 2013;2(4):355-361.

29. Quach S, Blais C, Quan H. Administrative data have high variation in validity for recording heart failure. Can J Cardiol. 2010;26(8):306-312. 
30. Statistique Canada. Population urbaine et rurale, par province et territoire (NouvelleÉcosse) [Internet]. Ottawa (Ont.) : Statistique Canada; 2011. Consultable en ligne à la page : http://www.statcan.gc.ca /tables-tableaux/sum-som/102/cst01/demo 62d-fra.htm

31. Marrie R, Fisk J, Stadnyk K et collab. The incidence and prevalence of multiple sclerosis in Nova Scotia, Canada. Can J Neurol Sci. 2013;40(6):824-831.

32. Bernstein CN, Blanchard JF, Rawsthorne P, Wajda A. Epidemiology of Crohn's disease and ulcerative colitis in a central Canadian Province: a population-based study. Amer J Epidemiol. 1999;149(10):916-924.

33. Lowe AM, Roy P-O, B.-Poulin M et collab. Epidemiology of Crohn's Disease in Quebec, Canada. Inflamm Bowel Dis. 2009;15(3):429-435.

34. Veuglers PJ, Fisk JD, Brown MG et collab. Disease progression among multiple sclerosis patients before and during a diseasemodifying drug program: a longitudinal population-based evaluation. Mult Scler. 2009;15:1286-294.

35. Klabunde CN, Warren JL, Legler JM. Assessing comorbidity using claims data: an overview. Med Care. 2002;40(8 Suppl): IV-26-35.

36. Landis JR, Koch GG. The measurement of observer agreement for categorical data. Biometrics. 1977;33(1):159-174.

37. Kisely S, Lin E, Gilbert C, Smith M, Campbell LA, Vasiliadis HM. Use of administrative data for the surveillance of mood and anxiety disorders. Aust N Z J Psychiatry. 2009;43(12):1118-1125.

38. Martens P, Fransoo R, McKeen N et collab. Patterns of regional mental illness disorder diagnoses and service use in Manitoba: a population-based study [Internet]. Winnipeg (Man.) : Manitoba Centre for Health Policy; 2004 [édition révisée du 18 nov. 2004]. PDF téléchargeable $(4,80 \mathrm{Mo})$ à partir du lien : http://mchp-appserv.cpe.umanitoba.ca /reference/mental.health.pdf

39. West SL, Richter A, Melfi CA, McNutt M, Nennstiel ME, Mauskopf JA. Assessing the Saskatchewan database for outcomes research studies of depression and its treatment. J Clin Epidemiol. 2000;53(8):823-831.
40. Tu K, Campbell NRC, Chen Z, Cauch-Dudek KJ, McAlister FA. Accuracy of administrative databases in identifying patients with hypertension. Open Medicine. 2007;1(1):e18-26.

41. Hux JE, Ivis F, Flintoft V, Bica A. Diabetes in Ontario: determination of prevalence and incidence using a validated administrative data algorithm. Diabetes Care. 2002;25(3): 512-516.

42. Reid AY, St Germaine-Smith C, Liu M et collab. Development and validation of a case definition for epilepsy for use with administrative health data. Epilepsy Res. 2012;102(3):173-179.

43. Elixhauser A, Steiner C, Harris DR, Coffey RM. Comorbidity measures for use with administrative data. Med Care. 1998;36(1): 8-27.

44. Wilchesky M, Tamblyn RM, Huang A. Validation of diagnostic codes within medical services claims. J Clin Epidemiol. 2004;57(2):131-141.

45. Luthi JC, Troillet N, Eisenring MC et collab. Administrative data outperformed singleday chart review for comorbidity measure. Int J Qual Health Care. 2007;19(4):225-231.

46. Bayliss EA, Ellis JL, Steiner JF. Subjective assessments of comorbidity correlate with quality of life health outcomes: initial validation of a comorbidity assessment instrument. Health and Quality of Life Outcomes. 2005;3:51. 